\author{
Bruno Lasserre, \\ Vice-président du Conseil d'État ${ }^{1}$
}

\title{
LE CONSEIL D'ÉTAT FACE À LA CRISE SANITAIRE DU COVID-19
}

UDK: $1.321: 616.2-036.21(44)$

DOI: $10.31141 /$ zrpfs.2021.58.140.401

Izvorni znanstveni rad

Primljeno: 15. 1. 2021.

\begin{abstract}
U radu autor analizira način na koji se Državno vijeće u Francuskoj bavilo zdravljem u okolnostima izvanrednih kriza. Iako su sudske metode i sredstva njegove kontrole blisko povezane s povijesti i kulturom zemlje autor je pozivajući se na Pascala ("Istina ispod Pirineja je negdje drugdje pogreška") uvjeren da ova institucija podržava ključnu vrlinu opće pravednosti. Također je uvjeren da unatoč svih razlika koje nas dijele, moramo učiti jedni od drugih, što i podiže vrijednost bilateralne suradnje.
\end{abstract}

Ključne riječi: Državno vijeće, zdravstvena kriza, pandemija, Covid 19

Split, Jeudi 17 septembre 2020

Monsieur le ministre [de la justice et de l'administration], Monsieur le président [du Haut tribunal administratif de la République de Croatie], Mesdames et Messieurs les juges, Monsieur le maire, Monsieur l'ambassadeur, Mesdames et Messieurs les professeurs, Mesdames et Messieurs, Chers amis,

Je suis heureux et honoré d'intervenir aujourd'hui pour la $14^{\text {ème }}$ édition des journées administratives et juridiques franco-croates. Ces journées constituent, depuis longtemps déjà, un événement important pour les relations entre la Croatie et la France. Elles réunissent chaque année l'élite administrative et universitaire croate pour dialoguer avec des juges et des professeurs français. Et nos échanges sont toujours extrêmement fructueux, tant sont nombreux les sujets qui traversent nos frontières nationales et sur lesquels nous avons à apprendre de l'expérience et des réflexions de nos homologues étrangers. Je peux d'ailleurs vous dire que parmi toutes les relations que le Conseil d'État entretient avec d'autres pays, les journées franco-croates sont un parfait exemple d'une coopération bilatérale qui fonctionne, d'une coopération bilatérale aussi amicale que fertile.

1 Texte écrit en collaboration avec Guillaume Halard, magistrat administratif, chargé de mission auprès du vice-président du Conseil d'État 
Le coronavirus n'a donc pas empêché que nous nous retrouvions : j'en suis d'autant plus ravi que beaucoup d'événements similaires ont été annulés, alors que l'organisation d'aujourd'hui démontre qu'il est possible de continuer à nous rencontrer tout en respectant strictement les règles de distanciation physique. En langage juridictionnel, on appelle cela « concilier le droit au dialogue et l'impératif de protection de la santé »... Nous verrons que ces exercices de conciliation ne sont pas toujours aisés, surtout en temps de crise, et c'est pourquoi je tiens à remercier très chaleureusement Neven Simac et Marc Gjidara, sans lesquels rien n'aurait été possible. Je tiens également à rendre hommage à deux de mes collègues, qui se sont impliqués depuis l'origine dans l'organisation de ces journées : Jean-Louis Dewost, qui nous a malheureusement quittés récemment, et Jean Massot.

Nous ne pouvons toutefois pas faire abstraction des circonstances très particulières dans lesquelles nous nous trouvons depuis plusieurs mois. Vous remarquerez en effet que cette édition des journées franco-croates a lieu dans une configuration inédite : exceptionnellement, l'université Paris-II n'est pas représentée dans la délégation française, ainsi réduite, que je suis seul à former avec Martine de Boisdeffre, présidente de la section du rapport et des études du Conseil d'État.

Surtout, la crise nous a fourni un thème tout trouvé pour ce colloque. Ses incidences administratives ont été et continuent d'être considérables : quasiment tous les pays touchés ont fait le choix de se doter de législations d'urgence et d'imposer des mesures de police sans précédent, que l'on pense à la fermeture de leurs frontières ou au confinement plus ou moins strict de leur population. Les administrations ont toutes dû s'adapter pour continuer à remplir leurs missions de service public en dépit des contraintes sanitaires. Et les incidences juridiques n'ont pas été moins importantes : même lorsqu'ils sont justifiés, les régimes d'exception et les lois de circonstances sont toujours problématiques ; ils font peser sur l'État de droit des menaces qu'on ne peut pas prendre à la légère. Ces menaces sont d'autant plus fortes que les gouvernements sont sommés d'agir en urgence - c'est-à-dire sans recul et à partir d'informations limitées - et que, comme ce fut le cas au pic de la pandémie, les assemblées parlementaires ne sont plus capables de jouer pleinement leur rôle de contre-pouvoir. Reste alors le pouvoir juridictionnel : la crise, tout du moins en France, a braqué les projecteurs sur le juge, vers qui se sont reportés beaucoup de citoyens inquiets, voire sceptiques vis-à-vis de l'action de l'exécutif. C'est à lui qu'est revenu la lourde tâche d'apprécier, au cas par cas, le bien-fondé des politiques gouvernementales mises en œuvre pour faire face à la crise.

Compte tenu de sa double fonction de conseiller juridique et de juge administratif suprême, le Conseil d'État s'est retrouvé au carrefour de toutes les tensions. Dans le cadre de sa fonction consultative, le Conseil d'État rend des avis indépendants sur les projets de loi, d'ordonnance et de textes règlementaires les plus importants : il a ainsi examiné tous les textes qui ont fondé la politique de crise et formulé des recommandations très souvent suivies par le gouvernement. Dans le cadre de ses fonction juridictionnelles, le Conseil d'État est à fois juge de cassation et, parfois, juge d'appel des décisions rendues par les tribunaux administratifs et les 
cours administratives d'appel, mais il est aussi, dans certains cas, juge de premier et dernier ressort, en particulier pour la plupart des actes réglementaires à portée nationale. Le nombre sans précédent de requêtes dont il a été saisi a ainsi fait du Conseil d'État le réceptacle de toutes les peurs et les frustrations générées par la crise, souvent légitimes, parfois teintées de militantisme, et le garant principal de la continuité de l'Etat de droit.

Même si les textes et les requêtes continuent d'abonder les greffes du contentieux et des sections consultatives, nous disposons aujourd'hui d'un certain recul pour réfléchir à la manière dont le Conseil d'État a rempli ses fonctions durant la crise. Je vous propose donc de décrire comment il s'est organisé pour continuer à remplir ses missions (II), le sens qu'il leur a donné et comment il a exercé ses pouvoirs, en particulier juridictionnels (III). Mais il me faut commencer par exposer le cadre juridique et les mesures prises par les pouvoirs publics pour faire face à la pandémie (I).

\section{Pour faire face à la crise sanitaire, un cadre juridique d'exception a été défini et de très nombreux textes ont été rédigés}

A. Pouvait-on gérer efficacement la crise dans le cadre juridique « normal » alors en vigueur ? Cette question se pose à vrai dire à chaque grande catastrophe, et elle ne manque jamais de susciter des débats sur les risques que recèlent les états d'exception pour les libertés. Le gouvernement et le Parlement ont toutefois préféré instituer un régime juridique particulier - l'état d'urgence sanitaire - afin de conférer à l'autorité administrative un cadre d'intervention clair et organisé

Dès que le Covid-19 a commencé à se propager sur le territoire français, le gouvernement et le Parlement, comme dans la plupart des autres pays, se sont retrouvés face au mur : il a fallu agir vite, prendre des mesures fortes et concrètes afin de protéger la santé de la population.

Deux cadres spécifiques étaient alors susceptibles de fonder leur action : le premier résultait d'une loi de $2004^{2}$, modifiée en $2007^{3}$ et censée offrir au gouvernement des outils pour faire face à une menace sanitaire de grande ampleur. C'est sur ce fondement que les premières mesures ont été prises dès la fin de mois de février 2020 : par plusieurs arrêtés, le ministre de la santé a notamment fermé un grand nombre d'établissements recevant du public ${ }^{4}$, interdit les rassemblements

2 Loi n $2004-806$ du 9 août 2004 relative à la politique de santé publique

3 Loi n ${ }^{\circ}$ 2007-294 du 5 mars 2007 relative à la préparation du système de santé à des menaces sanitaires de grande ampleur

4 Arrêté du 14 mars 2020 portant diverses mesures relatives à la lutte contre la propagation du virus covid-19 
de plus de 5000 personnes $^{5}$ et les déplacements de plus de 100 personnes $^{6}$, ou encore suspendu l'accueil des enfants dans les établissements scolaires ${ }^{7}$. Le second fondement juridique était, en dépit de son nom, plus classique : il s'agit de la théorie des circonstances exceptionnelles ${ }^{8}$, définie par le Conseil d'État à la fin de la Première Guerre mondiale et en vertu de laquelle l'autorité administrative peut exceptionnellement déroger aux règles normales de compétence, de forme et d'objet des actes administratifs lorsque les circonstances l'exigent absolument. C'est en combinant ce fondement à ses pouvoirs de police générale qu'immédiatement après que le Président de la République eut annoncé des restrictions fortes de déplacement, le Premier ministre a imposé un confinement consistant en l'interdiction pour toute personne de se déplacer hors de son domicile, sous réserve d'exceptions limitativement énumérées et devant être dûment justifiées?.

Ces bases juridiques sont toutefois vite apparues insuffisantes : en 2004 et 2007, le législateur était resté au milieu du gué en cantonnant le ministre de la santé à la seule action préventive ${ }^{10}$ quand la théorie des circonstances exceptionnelles, certes utile pour justifier des mesures ponctuelles, l'est en revanche beaucoup moins pour fonder une stratégie globale de lutte contre une pandémie aussi grave. Juridiquement risquée - car dépendant exclusivement de l'appréciation du juge -, elle ne convenait ni à la variété des mesures nécessitées par la crise, ni à leur caractère général et continu dans le temps. Elle ne convenait pas davantage à la garantie des libertés puisque les mesures susceptibles d'être autorisées ne font l'objet d'aucune détermination préalable et ne sont entourées d'aucune garantie procédurale ${ }^{11}$. Telles sont les principales raisons qui ont justifié le vote d'une loi instituant un nouvel «état d'urgence sanitaire ${ }^{12} »$. Une fois déclenché, il a sensiblement accru les pouvoirs de police administrative : les réquisitions de matériel médical, les mesures d'hygiènes et le confinement généralisé imposé sur ce nouveau fondement par le décret du 23 mars $2020^{13}$ sont de ce point de vue les mesures les plus spectaculaires prises dès l'entrée en vigueur de l'état d'urgence sanitaire.

L'insuffisance alléguée du droit existant et l'opportunité de définir un nouveau régime d'exception ont été largement débattus, au Parlement, en doctrine et dans les médias généralistes. Il faut à cet égard noter que la loi du 23 mars 2020 ne fait pas

5 Arrêté du 4 mars 2020 portant diverses mesures relatives à la lutte contre la propagation du virus covid-19

6 Arrêté du 13 mars 2020 portant diverses mesures relatives à la lutte contre la propagation du virus covid-19

7 Arrêté du 14 mars 2020 précité

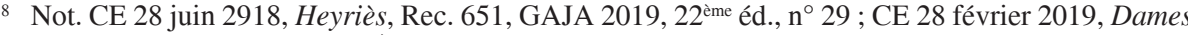

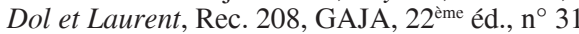

9 Décret $\mathrm{n}^{\circ} 2020-260$ du 16 mars 2020 portant réglementation des déplacements dans le cadre de la lutte contre la propagation du virus covid-19

${ }_{10} \mathrm{~V}^{\circ}$ J.-E. Gicquel, « Covid-19 : crise sanitaire et crise des normes », D. 2020.719

$11 \mathrm{~V}^{\circ}$ J. Petit, «L'état d'urgence sanitaire », AJDA 2020. 833

12 Loi n 2020-290 du 23 mars 2020 d'urgence pour faire face à l'épidémie de covid-19

13 Décret $n^{\circ}$ 2020-293 du 23 mars 2020 prescrivant les mesures générales nécessaires pour faire face à l>épidémie de covid-19 dans le cadre de l>état d>urgence sanitaire 
qu'accroître les pouvoirs de police administrative : elle encadre aussi de manière assez stricte l'action du gouvernement. Au-delà de deux mois, l'état d'urgence doit être prorogé par la loi ; le champ des mesures pouvant être décrétées est précisément délimité $^{14}$; les principes de nécessité et de proportionnalité sont expressément rappelés ${ }^{15}$; le Parlement doit être informé sans délai des mesures prises par le gouvernement ${ }^{16}$; les données scientifiques disponibles ayant motivé la décision de déclarer l'état d'urgence sanitaire doivent être rendues publiques ${ }^{17} \ldots$ Mentionnons enfin l'une des particularités les plus notables de cette loi, qui est d'avoir créé, pour la première fois ${ }^{18}$, un régime d'exception « expérimental »: le Parlement a en effet décidé d'en limiter la mise en œuvre à la gestion du Covid-1919 et de renvoyer à une évaluation pour le pérenniser le cas échéant ${ }^{20}$.

Il est certain que la loi a permis de clarifier et d'ordonner le cadre juridique de gestion de la crise. Mais sans doute est-ce sage, dans le même temps, de s'être contraint à le repenser une fois passée la tempête.

\section{B. Tout un train de mesures a par ailleurs été pris, notamment par ordonnances, pour adapter l'organisation administrative, économique et sociale du pays}

A côté des mesures de police visant principalement à lutter contre la propagation de l'épidémie, le gouvernement a cherché à adapter aux circonstances les règles régissant tout un ensemble d'activités.

Près d'une quarantaine de lois, notamment de finances, ont été votées.

Mais la lourdeur de la procédure législative couplée à la difficulté, pour les parlementaires, de se réunir explique que la législation déléguée - qui permet au gouvernement, dûment habilité par le Parlement, d'intervenir dans le domaine de la loi - ait été préférée à la loi. Pendant la crise, le recours aux ordonnances, souvent

14 Art. 2 de la loi n 2020-290 du 23 mars 2020

15 Ibid. («Les mesures prescrites en application des $1^{\circ}$ à $10^{\circ}$ du présent article sont strictement proportionnées aux risques sanitaires encourus et appropriées aux circonstances de temps et de lieu. Il y est mis fin sans délai lorsqu>elles ne sont plus nécessaires. »)

16 Ibid. (« L>Assemblée nationale et le Sénat sont informés sans délai des mesures prises par le Gouvernement au titre de l>état d`urgence sanitaire. L〉Assemblée nationale et le Sénat peuvent requérir toute information complémentaire dans le cadre du contrôle et de l>évaluation de ces mesures. »)

17 Ibid. («L〉état d〉urgence sanitaire est déclaré par décret en conseil des ministres pris sur le rapport du ministre chargé de la santé. Ce décret motivé détermine la ou les circonscriptions territoriales à l>intérieur desquelles il entre en vigueur et reçoit application. Les données scientifiques disponibles sur la situation sanitaire qui ont motivé la décision sont rendues publiques. »)

18 Elle diffère en cela des autres régimes d'exception que fondent par exemple la loi $\mathrm{n}^{\circ} 55-585$ du 3 avril 1955 et les article 16 et 36 de la Constitution.

19 L'article 7 de la loi dispose en effet que « Le chapitre Ier bis du titre III du livre Ier de la troisième partie du code de la santé publique est applicable jusqu'au 1er avril 2021. »

20 « Il appartiendra au Parlement de dresser un bilan de l>application du dispositif et, si son utilité est établie, de le pérenniser, le cas échéant, modifié au regard des premiers mois d`expérience » (P. Bas, Rapp. fait au nom de la commission des lois du Sénat, $n^{\circ} 381$, p. 25) 
pointé du doigt comme le symptôme d'un « dérèglement ${ }^{21}$ » constitutionnel et d'une « crise de la fonction législative $»^{22}$, s'est ainsi imposé par son efficacité. Plus de 70 ordonnances ont ainsi été prises dans les domaines les plus variés : travail et politiques sociales avec, par exemple, l'institution d'un dispositif de chômage partiel ${ }^{23}$ et la prolongation des droits sociaux ${ }^{24}$, éducation ${ }^{25}$, culture et sport ${ }^{26}$, santée ${ }^{27}$ ou encore banques ${ }^{28}$ et entreprises ${ }^{29}$.

Enfin, lorsque la Constitution le permettait, le gouvernement a agi par décret.

La nature des textes utilisés pour faire face à la crise n'est pas sans incidence sur le rôle du Conseil d'État, en particulier en tant que juge. Le Conseil d'État n'est en effet traditionnellement pas le juge de la loi : elle ne peut être directement attaquée devant lui, il n'en contrôle pas la constitutionnalité ${ }^{30}$ et ne vérifie sa conformité aux engagements internationaux de la France que de manière indirecte ${ }^{31}$. En revanche, il est juge des ordonnances tant qu'elles n'ont pas été ratifiées : jusqu'à cette date, celles-ci revêtent le caractère d'actes administratifs et sont donc susceptibles de faire l'objet d'un recours devant le juge administratif ${ }^{32}$. Puisque le Conseil constitutionnel ne dispose pas de procédures d'urgence et n'était donc pas en mesure de contrôler avec la célérité requise les lois promulguées durant la crise, le recours aux ordonnances a paradoxalement été mieux à même de garantir l'accès au juge. Nous verrons à cet égard que de nombreux contentieux se sont noués autour d'ordonnances.

21 P. Delvolvé, «L'été des ordonnances », RDFA, 2005, p. 909

22 J.-M. Sauvé, « La législation déléguée », Intervention du 6 juin 2014 à l'occasion du colloque organisé par le Centre d'études constitutionnelles et politiques, l'Institut Cujas et la Société de législation comparée (en ligne : https://www.conseil-etat.fr/actualites/discours-et-interventions/la-legislationdeleguee\#_ftn26)

23 Par exemple : ordonnance $n^{\circ}$ 2020-346 du 27 mars 2020 portant mesures d'urgence en matière d'activité partielle

24 Par exemple : ordonnance $\mathrm{n}^{\circ}$ 2020-312 du 25 mars 2020 relative à la prolongation de droits sociaux

25 Par exemple : ordonnance $n^{\circ} 2020-351$ du 27 mars 2020 relative à l'organisation des examens et concours pendant la crise sanitaire née de l'épidémie de covid-19

26 Par exemple : ordonnance $n^{\circ}$ 2020-538 du 7 mai 2020 relatif aux conditions financières de résolution de certains contrats en cas de force majeure dans les secteurs de la culture et du sport

27 Par exemple : ordonnance $\mathrm{n}^{\circ} 2020-309$ du 25 mars 2020 relative à la garantie de financement des établissements de santé et aux régimes complémentaires obligatoires de sécurité sociale

28 Par exemple : ordonnance $n^{\circ} 2020-534$ du 7 mai 2020 portant diverses dispositions en matière bancaire

29 Par exemple : ordonnance $\mathrm{n}^{\circ} 2020-740$ du 17 juin 2020 relative à l'octroi d'avances en compte courant aux entreprises en difficulté par les organismes de placement collectif de capital investissement et les sociétés de capital-risque

30 CE 6 novembre 1936, Sieur Arrighi, Rec. P. 966 ; CE 5 janvier 2005, Deprez et Baillard, $\mathrm{n}^{\circ}$ 275341 et 275534

$31 \mathrm{~V}^{\circ}$ CE 22 janvier 2013, Confédération générale des cadres - Centrale (CGC-Centrale) et Confédération générale des cadres de la direction générale des finances publiques (CGC-DGFIP), $\mathrm{n}^{\circ}$ 348209

$32 V^{\circ} \mathrm{CE} 13$ juin 2013, Conseil national de l'ordre des infirmiers et autres, $\mathrm{n}^{\circ} 408325$ et $\mathrm{s}$. 


\section{Le Conseil d'État s'est lui aussi organisé pour assurer la continuité de ses missions essentielles, ce qui lui a permis d'examiner en urgence tous les projets de textes qui lui ont été soumis et de juger un nombre exceptionnel de requêtes}

\section{A. Immédiatement après le début de la crise, le Conseil d'État a activé son plan de continuité d'activité et mis à profit ses outils numériques}

Dès l'annonce des premières consignes gouvernementales, début mars, le Conseil d'État a activé son plan de continuité d'activité. Ce plan avait été élaboré quelques mois auparavant en prévision non pas d'une pandémie, mais d'une crue exceptionnelle de la Seine... Il identifiait des missions « essentielles » - en particulier le jugement des requêtes urgentes et le conseil au gouvernement - ainsi que les personnels nécessaires pour les remplir. Ce plan s'est donc révélé très utile : il nous a permis d'agir vite et de manière coordonnée.

Le télétravail est immédiatement devenu le principe pour la grande majorité des agents et des membres du Conseil d'État. Les outils numériques, dans lesquels nous avions investi depuis plusieurs années, ont été pour cela d'une grande aide : chaque personnel a pu disposer, depuis chez lui, d'un accès aux réseaux partagés et aux bases de données dont nous nous servons quotidiennement. La direction des services d'information a par ailleurs développé un système de visio-conférence, encore embryonnaire lorsque la crise a commencé, mais rendu rapidement opérationnel pour les services comme pour les sections. C'est ainsi par exemple grâce à la visioconférence que, le 27 avril, notre rapport d'activité a été approuvé avant d'être diffusé par la section du rapport et des études. À noter que la solution de visio-conférence que nous avons choisie comprend un mécanisme de cryptage des données et toutes les données recueillies sont hébergées sur des serveurs physiquement présents dans nos locaux : ceci est important car beaucoup des travaux du Conseil d'État sont couverts par le secret.

\section{B. Les sections administratives sont parvenues à rendre un très grand nombre d'avis dans des délais serrés}

Dans les sections administratives, tous les membres et agents ont travaillé de chez eux et toutes les séances de travail se sont tenues par visio-conférence. Les sections se sont d'abord réunies en formations restreintes (président, présidentadjoint et rapporteur) ; ces formations ont ensuite été progressivement élargies à mesure que les contraintes sanitaires se desserraient. La commission permanente s'est elle aussi régulièrement réunie à distance et l'Assemblée générale a délibéré par voie électronique sur le rapport d'activité. La principale difficulté rencontrée par les sections administratives a été la communication avec les commissaires du gouvernement, dont les outils de visio-conférence répondaient à des normes de sécurité non compatibles avec celles du Conseil d'État : ils ont pu contourner ce 
problème en recourant à la téléconférence ou en se connectant via leurs ordinateurs personnels.

Les sections ont ainsi pu examiner tous les projets de textes produits par le gouvernement : au total, entre le 12 mars et le 30 juin 2020, 256 projets de textes législatifs et réglementaires ont été examinés, dont 232 étaient en lien avec la crise sanitaire. C'est environ $25 \%$ de plus qu'en temps normal ${ }^{33}$, étant entendu par ailleurs que les délais d'instruction dont a disposé le Conseil d'État pour ces textes étaient particulièrement courts.

La plupart des avis rendus pendant la crise n'ont pas été publiés ${ }^{34}$. Il ne m'est donc pas permis d'évoquer leur teneur, mais je peux vous dire que le Conseil d'État a scrupuleusement veillé, comme c'est son habitude, à améliorer la qualité des normes produites, leur cohérence et leur efficacité, ainsi qu'à garantir leur régularité juridique, notamment leur conformité à la Constitution et aux engagements internationaux de la France. Je peux également vous dire que le gouvernement a fréquemment suivi les avis rendus par le Conseil d'État, qui étaient d'autant plus utiles que le travail gouvernemental était lui aussi réalisé dans des conditions exceptionnellement difficiles.

\section{Les juges de la section du contentieux ont quant à eux traité un nombre sans précédent de requêtes tout en préparant la sortie de crise}

A la section du contentieux, la mission essentielle définie dans le plan de continuité était de juger toutes les affaires urgentes. Pour ce faire, une équipe de quinze juges expérimentés, réunis autour du président et des présidents-adjoints de la section, a été constituée : même si les procédures de référé sont traditionnellement des procédures à juge unique, c'est bien comme une équipe qu'ont travaillé ces juges qui n'ont cessé de se coordonner et de discuter des questions qu'ils avaient à trancher. Les audiences se sont toutes tenues au Palais-Royal, en présence des juges ; les parties, quant à elles, y ont souvent assisté par visio-conférence, ce qu'une ordonnance du 25 mars $2020^{35}$ a exceptionnellement autorisé. Le Conseil d'État a tout fait pour ne pas déroger au principe fondamental selon lequel les « débats ont lieu en audience publique ${ }^{36} »$ : les audiences se sont tenues portes ouvertes ; le grand public - au demeurant confiné - n'a pas été autorisé à se s'y rendre, mais la presse y a systématiquement été conviée et beaucoup de journalistes sont venus au

33 Entre le 12 mars et le 21 juin 2019, 206 projets de textes législatifs et réglementaires avaient été examinés par les sections consultatives du Conseil d'État.

34 Seuls les avis rendus sur des projets de loi sont systématiquement publiés, en vertu d'une coutume qui s'est installée depuis une décision du président François Hollande (Discours du Président de la République, mardi 20 janvier 2015, à l'occasion des vœux au Parlement et aux corps constitués).

35 Article 7 de l'ordonnance $\mathrm{n}^{\circ} 2020-305$ du 25 mars 2020 portant adaptation des règles applicables devant les juridictions de l'ordre administratif, modifié par l'article $1^{\text {er }}$ de l'ordonnance $n^{\circ} 2020-558$ du $1 »$ mai 2020.

36 Article L. 6 du code de justice administrative 
Palais-Royal. L'écho médiatique des travaux du Conseil d'État n'a d'ailleurs jamais été aussi large.

Cette organisation a permis aux juges de traiter un nombre exceptionnel de requêtes. Le Conseil d'État ne s'attendait pas à être tant sollicité : entre le 12 mars et la fin du mois de juin, la section du contentieux a enregistré 382 requêtes en lien avec le Covid-19, dont 230 en référé, c'est-à-dire à juger en urgence. C'est $60 \%$ de plus qu'en période normale ${ }^{37}$ ! Toutes ces affaires ont été jugées dans les plus brefs délais, souvent moins de 48 heures. Il convient ici de préciser que les règles de procédures prévoient différentes voies pour traiter les requêtes en référé : la voie normale est celle de l'audience après instruction ; le code de justice administrative prévoit par ailleurs, depuis l'institution des référés administratifs, la possibilité pour le juge de rejeter par ordonnance une demande lorsqu'elle ne présente pas un caractère d'urgence ou qu'il apparaît manifeste, au vu de la demande, qu'elle ne relève pas de la compétence de la juridiction administrative, est irrecevable ou mal fondée ${ }^{38}$. L'ordonnance du 25 mars 2020, qui a adapté les règles applicables devant la juridiction administrative le temps de la crise, a enfin ouvert une troisième option : lorsque le juge estime, après avoir instruit l'affaire, qu'une audience n'est pas nécessaire, il peut décider de s'en $\operatorname{passer}^{39}$. Cette dérogation aux règles traditionnelles était tout à fait justifiée pendant la crise, dès lors que le principe de publicité des débats devait être concilié avec l'objectif, contradictoire, de lutte contre la propagation de l'épidémie. Et les chiffres montrent que le Conseil d'État n'a pas fait un usage excessif de ces voies alternatives : sur les 230 dossiers que je viens de mentionner, environ $60 \%$ ont fait l'objet d'une instruction et $35 \%$ d'une audience.

La section du contentieux ne s'est toutefois pas contentée de traiter les affaires urgentes : les présidents de chambres ont tous souhaité anticiper la sortie de crise en continuant l'instruction des dossiers pendants. Les chambres ont donc continué à travailler normalement, à deux réserves près : les séances d'instruction ont eu lieu par visio-conférence et les audiences sur les affaires «non urgentes » ont été différées. A la fin du confinement, la section du contentieux disposait ainsi d'un stock considérable d'affaires en état d'être jugées. Dès le 13 mai, les audiences ont repris à un rythme soutenu afin de sortir au plus vite ce surplus de dossiers.

37 Entre le 12 mars et le 25 juin 2019, 141 requêtes en référés avaient été enregistrées à la section du contentieux

38 Article L. 522-3 du code de justice administrative

39 Article 9 de l'ordonnance $\mathrm{n}^{\circ} 2020-305$ du 25 mars 2020 portant adaptation des règles applicables devant les juridictions de l'ordre administratif 


\section{Face à la diversité des requêtes qui lui ont été adressées, le Conseil d'État est resté constant dans son contrôle : il a veillé à concilier les objectifs sanitaires poursuivis par le gouvernement avec la protection des droits et libertés, et toujours cherché à offrir des solutions concrètes et réalistes}

\section{A. La diversité et l'ampleur des questions posées au Conseil d'État ont été tout aussi exceptionnelles que leur nombre}

Avec le recul que nous avons aujourd'hui, on est en effet frappé par l'extrême diversité, à tous les points de vue, des requêtes adressées au Conseil d'Etat pendant la crise : elles ont émané de particuliers, d'entreprises, d'associations, de syndicats, de partis politiques ; certaines visaient à accroître la sévérité du confinement, d'autres à l'atténuer ; certaines posaient des questions de légalité purement juridiques, d'autres des problèmes extrêmement concrets relatifs, notamment, à l'organisation et au fonctionnement d'établissements et services publics ; la plupart des grandes libertés fondamentales ont été invoquées, du droit à la vie à la liberté d'aller et venir, en passant pas le droit à la santé, la liberté de culte, la liberté de réunion, la liberté d'entreprendre ou encore le droit de manifester.

On peut identifier dans cette masse trois grandes catégories de demandes.

La première est la plus classique : elle regroupe les demandes tendant à la suspension d'actes juridiques pris pour faire face à l'épidémie. Il a pu s'agir de mesures de police administrative : la Fédération nationale des marchés de France a ainsi contesté la décision du gouvernement de fermer les marchés alimentaires ${ }^{40}$, et la Fédération française des usagers de la bicyclette l'interdiction faite aux cyclistes de se déplacer durant le confinement ${ }^{41}$. Après le pic de la crise, des contentieux se sont également noués autour de l'encadrement des libertés de cultes ${ }^{42}$ et de manifestation ${ }^{43}$, justifié par la nécessité de respecter les règles de distanciation physique. Notons que de manière assez inédite, la première requête déposée devant le Conseil d'État n'avait pas pour objectif, comme c'est traditionnellement le cas dans le contentieux de la police, d'atténuer une restriction de liberté imposée au nom d'un intérêt supérieur - ordre public généralement, droits à la santé et au respect de la vie pendant cette pandémie -, mais au contraire d'accroître ces restrictions en imposant des modalités de confinement encore plus strictes que celles qui avaient été décidées par le gouvernement ${ }^{44}$. Enfin, par la nature des questions qu'elles posaient, les requêtes réclamant la suspension des dispositions d'ordonnances

40 CE, ord., $1^{\mathrm{er}}$ avril 2020, Fédération nationale des marchés de France, ${ }^{\circ} 439762$

41 CE, ord., 30 avril 2020, Fédération française des usagers de la bicyclette, $\mathrm{n}^{\circ} 440179$

42 CE, ord., 18 mai 2020, M. W. et autres, $\mathrm{n}^{\circ}$ 440366, 440380, 440410, 440531, 440550, 440562, 440563, 440590

43 CE, ord., 13 juin 2020, Ligue des droits de l'homme et autres, $n^{\circ} 440846,440856,441015$

44 CE, ord., 22 mars 2020, Syndicat jeunes médecins, n 439674 
modifiant certaines règles de procédure devant les juridictions administratives ${ }^{45}$ ou les modalités de prolongation des détentions provisoires ${ }^{46}$ entrent dans cette première catégorie.

Dans une deuxième catégorie figurent les demandes qui tendaient à adapter l'organisation du service public afin de mieux protéger ses personnels ou ses usagers. Ce sont ici des carences administratives qui étaient alléguées, les requérants demandant au juge d'y remédier en identifiant, le cas échéant, les mesures d'organisation nécessaires. La mise à disposition de masques, gels et autres matériels de protection contre le virus a ainsi été réclamée pour les personnels de santé $^{47}$ ou dans les prisons ${ }^{48}$. Le problème des prisons illustre d'ailleurs la nature - et la difficulté - des questions qui ont pu être posées au juge, puisqu'en plus de la distribution de matériels, les requérants demandaient que tout un ensemble de procédures et de règles d'organisation soient adaptées afin de limiter la propagation du virus et de mieux prendre en charge les personnes malades : le prétoire du juge s'est subrepticement transformé en cabinet d'audit. Il a passé à l'examen, par exemple, l'opportunité de maintenir un régime de détention « portes ouvertes » ou les mesures d'hygiène applicables dans les cuisines. Dans un registre similaire, il lui a fallu vérifier que les aménagements d'un centre de rétention administrative permettaient que les étrangers retenus contaminés par le virus y demeurent et ne soient pas libérés ${ }^{49}$. Des associations ont également demandé au juge d'enjoindre au ministre de l'intérieur d'assurer l'enregistrement des demandes d'asile, ce que le Conseil d'État a fait après avoir vérifié qu'il était effectivement possible de mobiliser un minimum d'agents dans les services de la préfecture d'Ile-de-France ${ }^{50}$. On peut par ailleurs citer les requêtes tendant à organiser un meilleur dépistage ${ }^{51}$ et un meilleur accès aux soins ${ }^{52}$ pour les pensionnaires d'établissements hébergeant des personnes âgées, ou celles visant à améliorer la prise en charge des sans-abris ${ }^{53}$ pendant le confinement.

Une troisième catégorie comprend enfin les requêtes remettant en cause les grandes orientations de la politique sanitaire du gouvernement. Je pense ici à aux requêtes demandant au juge d'enjoindre au gouvernement d'autoriser la prescription

45 CE, ord., 8 juin 2020, Association Elena France et autres, ${ }^{\circ} 440717,440812,440867$ 439898

CE, ord., 3 avril 2020, Union des jeunes avocats de Paris et autres, , 439877, 439887, 439890,

47 CE, ord., 28 mars 2020, Mme A. et autres, n 439693

48 Pour les personnels pénitentiaires : CE, ORD., 8 avril 2020, Syndicat national pénitentiaire FO personnels de surveillance, $\mathrm{n}^{\circ} 439821$; pour les détenus : CE, ORD., 8 avril 2020, Section française de l'OIP et autres, $\mathrm{n}^{\circ} 439827$

49 CE, ord., 7 mai 2020, Ministre de l'intérieur c. Association « Avocats pour la défense des droits des étrangers » et autres, $\mathrm{n}^{\circ} 440255$

50 CE, ord., 30 avril 2020, Ministre de l'intérieur, $\mathrm{n}^{\circ} 440250,440253$

51 CE, ord., 15 avril 2020, Union nationale des syndicats Force ouvrière santé privée et autres, $\mathrm{n}^{\circ}$ 440002

52 CE, ord., 15 avril 2020, Association Coronavictimes et autres, ${ }^{\circ} 439910$

53 CE, ord., 2 avril 2020, Fédération nationale du droit au logement et autres, $\mathrm{n}^{\circ} 439763$ 
d'hydroxy-chloroquine ${ }^{54}$, de modifier la liste des entreprises considérées comme « essentielles à la Nation ${ }^{55}$, ou encore de réquisitionner des stocks ${ }^{56}$ ou des entreprises ${ }^{57}$ pour assurer un approvisionnement suffisant de matériels sanitaires. Certains requérants se sont même présentés devant le juge avec une stratégie globale, déclinées en force mesures précises, de lutte contre l'épidémie en lui demandant d'ordonner au gouvernement de l'adopter ${ }^{58}$.

\section{B. Les réponses apportées à ces requêtes par le Conseil d'État doivent être lues à la lumière de l'office du juge des référés et témoignent de sa volonté d'offrir des solutions immédiates et concrètes aux atteintes qu'il constate}

Si le juge administratif a pu être tant sollicité durant la crise, c'est grâce aux procédures d'urgence dont il dispose depuis maintenant trente ans ${ }^{59}$ et qui ont montré, une nouvelle fois, toute leur efficacité.

La première est le référé-suspension, qui permet au juge de suspendre en urgence une décision administrative lorsqu'existe un doute sérieux sur sa légalitée ${ }^{60}$ : les pouvoirs du juge sont limités, il faut qu'existe une « décision », la loi ne prévoit pas expressément le délai dans lequel il doit statuer, mais le test auquel il se livre est relativement aisé à passer. Cette procédure est particulièrement efficace pour soumettre au juge des questions de pure légalité : c'est sur ce fondement, par exemple, que le Conseil d'État a suspendu les dispositions d'une ordonnance prise dans le cadre de l'état d'urgence sanitaire qui prévoyaient l'intervention d'un juge unique - et non plus, comme c'est le principe, d'une formation collégiale - pour statuer sur tous les recours déposés devant la Cour nationale du droit d'asile ${ }^{61}$. Le juge a en effet considéré que les difficultés de fonctionnement de cette Cour causées par l'épidémie n'étaient pas suffisantes pour justifier une dérogation aussi générale et systématique au principe de la collégialité.

Mais la procédure la plus utilisée a été celle du référé-liberté, dont l'équilibre est sensiblement différent de celui du référé-suspension : le juge du référé-liberté peut en effet ordonner toutes mesures nécessaires pour mettre fin à une atteinte grave et manifestement illégale portée par une personne publique à une liberté fondamentale ${ }^{62}$. Ses pouvoirs sont ainsi virtuellement illimités ${ }^{63}$; il n'a pas besoin d'être saisi d'une

54 CE, ord., 28 mars $2020, M$. A. et autres, $n^{\circ} 439765$

55 CE, ord., 18 avril 2020, Fédération des travailleurs de la métallurgie CGT, n ${ }^{\circ} 440012$

56 CE, ord., 22 mai 2020, Syndicats des jeunes médecins, $\mathrm{n}^{\circ} 440321$

57 CE, ord., 29 mars 2020, Debout la France, n 439798

58 CE, ord., 4 avril 2020, Mme Escolano et autres, $\mathrm{n}^{\circ} 439816$

59 Loi n ${ }^{\circ}$ 2000-597 du 30 juin 2000 relative au référé devant les juridictions administratives

${ }^{60}$ Article L. 521-1 du code de justice administrative

61 CE, ord., 8 juin 2020, Association Elena France et autres, préc.

62 Article L. 521-2 du code de justice administrative

${ }^{63}$ Le rapport du Groupe de travail de travail sur les procédures d'urgence entendait en effet permettre au juge d'exercer des « pouvoirs exorbitants du droit commun ». 
« décision » et peut s'emparer de tous comportements de l'administration, action ou inaction ; il est enfin tenu d'agir en $48 \mathrm{~h}$. En contrepartie, la loi est particulièrement exigeante quant à l'atteinte qui doit être constatée.

Qu'il soit juge du référé-suspension ou du référé-liberté, le Conseil d'État a fréquemment été conduit, durant la crise, à passer au crible du contrôle de proportionnalité les décisions ou carences contestées par les requérants. Cette mise en balance explique qu'au pic de l'épidémie, lorsque le risque sanitaire était maximum, le juge ait estimé que les restrictions aux libertés d'aller et venir, de réunion ou encore d'exercer une activité économique imposées par le gouvernement étaient nécessaires et proportionnées à l'objectif de protection de la santé de la population ${ }^{64}$. A l'inverse, lorsque le risque a commencé à décroître, certaines de ces restrictions ont perdu leur justification : c'est le sens de la série de décisions rendues lors de la phase de «déconfinement», par lesquelles le Conseil d'État a par exemple suspendu les interdictions de se rassembler dans les lieux de culte ${ }^{65}$ ou de manifester ${ }^{66}$ décidées par le gouvernement. Plus récemment, le juge des référés a jugé que les pouvoirs publics pouvaient rendre obligatoires le port du masque sur l'ensemble d'une commune, précisant toutefois que la délimitation des périmètres concernés devait être strictement justifiée par l'existence de zones à fort risque de contamination ${ }^{67}$.

Mais au-delà de ce contrôle de proportionnalité tout à fait classique, beaucoup des décisions rendues par le Conseil d'État ne peuvent se comprendre qu'à l'aune de l'office particulier du juge du référé-liberté. Ce juge est tout tourné vers la recherche des mesures permettant de mettre effectivement fin, de manière immédiate, aux atteintes à des libertés fondamentales qu'il constate ${ }^{68}$. C'est la raison pour laquelle le juge du référé-liberté tient compte des moyens dont dispose l'administration et des mesures qu'elle a déjà prises pour apprécier le caractère manifestement illégal de l'atteinte alléguée ${ }^{69}$. La nécessité de prendre en compte les moyens dont dispose l'administration a pu être questionnée ${ }^{70}:$ je crois pour ma part qu'elle est l'expression d'un principe de réalité que le juge ne peut pas se permettre d'ignorer : à quoi cela pourrait-il servir d'ordonner la distribution de milliers de masques, comme l'ont demandé certains requérants, alors que l'administration n'en était matériellement pas capable ? Elle témoigne aussi de l'humilité du juge du référé-liberté qui, statuant

${ }^{64}$ Par exemple : CE, ord., 24 mars 2020, M. et Mme Geffe, $\mathrm{n}^{\circ} 439695$ et CE, ord., 16 avril 2020, Mme Grisard Martel, $\mathrm{n}^{\circ} 440060$

65 CE, ord., 18 mai 2020, M. W. et autres, préc.

66 CE, ord., 13 juin 2020, Ligue des droits de l'homme et autres, préc.

67 CE, ord., 6 septembre 2020, Ministre des solidarités et de la santé, $\mathrm{n}^{\circ} 443750$ et 443751 (deux décisions)

$68 \mathrm{~V}^{\circ}$ not. CE, sect., 16 novembre 2011, Ville de Paris, Société d'économie mixte PariSeine, $\mathrm{n}^{\circ}$ 353172 ; et le considérant devenu classique : « le juge des référés peut ordonner à l'autorité compétente de prendre, à titre provisoire, des mesures d'organisation des services placés sous son autorité, dès lors qu'il s'agit de mesures d'urgence qui lui apparaissent nécessaires pour sauvegarder, à très bref délai, la liberté fondamentale à laquelle il est gravement, et de façon manifestement illégale, porté atteinte. »

69 «Le caractère manifestement illégal de l'atteinte doit s'apprécier notamment en tenant compte des moyens dont dispose l'autorité administrative compétente et des mesures qu'elle a déjà prises. »

$70 \mathrm{~V}^{\circ}$ not. C. Malverti et C. Beaufils, « Le référé en liberté », AJDA 2020. 1154 
en quarante-huit heures, n'a souvent pas les moyens de définir lui-même les mesures que l'administration devrait prendre en plusieurs semaines, ou même mois... La prise en compte des moyens dont dispose l'administration à la date à laquelle le juge statue était d'autant plus nécessaire durant la crise que beaucoup des contentieux portaient au fond sur la manière dont le gouvernement gérait une certaine rareté : rareté de matériels sanitaires, saturation des lits de réanimation, disponibilité des personnels.

Ceci étant dit, le juge du référé-liberté ne s'est jamais résigné à constater l'absence de moyens de l'administration, et il a constamment cherché les solutions concrètes de nature à mieux sauvegarder les libertés fondamentales invoquées par les requérants. Les audiences se sont à cet effet révélées extrêmement utiles : c'est très souvent grâce à la discussion orale avec les parties que des solutions ont été trouvées. Plus les contentieux mettaient en cause les modalité concrètes d'organisation et de fonctionnement de tel ou tel service public, plus la discussion est apparue utile au juge pour saisir tous les tenants et les aboutissants des problèmes qui lui étaient soumis. L'affaire relative aux mesures sanitaires pour les détenus est à cet égard topique : à l'issue d'une audience qui a duré plus de six heures, durant laquelle toutes les mesures déjà prises par l'administration pénitentiaire ont été scrutées avec attention, des mesures supplémentaires d'hygiène devant être appliquées dans les cuisines et un protocole permettant le signalement et la détection des cas symptomatiques ont été définis et l'administration s'est engagée à les mettre en œuvre sans délai ${ }^{71}$. Tenant compte de cet engagement, le juge a par conséquent rejeté la requête, mais ce rejet ne signifiait nullement que les demandes des requérants étaient infondées : elle voulait dire au contraire qu'à la date à laquelle le juge statuait, une solution satisfaisante au regard des moyens dont disposait l'administration avait été trouvée. Et bien entendu, dans les rares cas où l'administration n'a pas tenu les engagements qu'elle avait pris à l'audience, le juge des référés n'a pas manqué de la sanctionner ${ }^{72}$.

\section{Ce qu'on appelle dorénavant le « contentieux Covid » a mis en lumière le rôle essentiel que joue le Conseil d'État au service de l'État de droit mais aussi l'importance, pour lui, de ne pas sortir du cadre de ses pouvoirs}

Tout au long de la crise, le Conseil d'État a été présent, il a été en mesure d'instruire et de juger les requêtes qui lui ont été adressées, d'entendre les parties, de contrôler minutieusement l'action de l'administration. Grâce à ses procédures de référés, il a été l'interlocuteur privilégié, si ce n'est l'unique interlocuteur, de tous les citoyens qui ont souhaité remettre en cause certains aspects de la gestion de l'épidémie. Il a par ailleurs constamment mis sous tension l'administration, en particulier lors des audiences, au cours desquelles il a toujours cherché les moyens

71 CE, ord., 8 avril 2020, Section française de l'OIP, préc.

${ }_{72}$ CE, ord., 30 avr. 2020, Ministre de l'intérieur, Office français de l'immigration et de l'intégration, préc. 
de faire mieux, de moins attenter aux libertés fondamentales tout en garantissant l'efficacité de la lutte contre le virus. Il n'y a enfin pas eu de justice d'exception, ce qui est bien le signe d'une forme de continuité de l'État de droit malgré les circonstances : les procédures et les méthodes de contrôle du juge administratif n'ont pas varié, leur pertinence et leur souplesse sont apparues clairement.

Ceci étant dit, le juge s'est aussi trouvé au cœur d'attentes souvent contradictoires, auxquelles il a pris garde de répondre en restant conscient de la place qu'il occupe dans les institutions. Il a en particulier veillé à ne pas empiéter sur les domaines politiques et scientifiques. Comment aurait-il pu faire droit aux requêtes lui demandant d'ordonner au gouvernement d'autoriser la prescription d'hydroxy-chloroquine en médecine de ville alors que les études disponibles à cette date souffraient d'insuffisances méthodologiques et ne permettaient nullement de conclure à l'efficacité de ce produit ? Il ne revient pas au juge de parler à la place des scientifiques et seul le pouvoir politique aurait pu, le cas échéant, prendre le risque d'autoriser la prescription en dépit des incertitudes. De la même manière, lorsque le syndicat Jeunes médecins a demandé au juge des référés d'ordonner un confinement plus strict encore de l'ensemble de la population française, je comprends leurs inquiétudes tout à fait légitimes, mais je doute qu'un juge, et plus encore un juge de l'urgence, soit bien placé pour faire cela. Une telle décision est si lourde de conséquences, elle implique de prendre en compte tant d'intérêts différents qu'elle ne peut raisonnablement relever que de la stratégie sanitaire incombant au pouvoir politique. Ce que pouvait faire le juge - et c'est ce qu'il a fait - c'était d'identifier certaines incohérences dans le dispositif du gouvernement et de lui demander d'y remédier : il a ainsi enjoint au Premier ministre de préciser la portée de plusieurs dérogations à l'interdiction de se déplacer hors de son domicile - les « déplacements pour motifs de santé » et ceux « liés à l'exercice physique individuel » ${ }^{73}$. Je crois par ailleurs que pour le juge, rester sur cette ligne de crête entre politique et scientifique, conscient de ses pouvoirs et de ses limites, était d'autant plus délicat durant la crise que le droit - la loi, les règlements - ne prévoit pas précisément comment contrer une épidémie et allouer des ressources rares. Les notions de droit à la vie et de droit à la santé systématiquement mobilisées, aussi symboliques soient-elles, sont quant à elles loin d'être univoques, surtout quand le droit à la vie de certains doit être concilié avec celui de tous. Dernier écueil, enfin, auquel le juge doit faire attention : c'est celui de voir son prétoire se transformer en perchoir ${ }^{74}$, comme en attestent les prises de positions qui, pendant l'état d'urgence sanitaire, ont invité le juge des référés à substituer son contrôle à celui du Parlement défaillant ${ }^{75}$ ou les requêtes fantaisistes - car il y en a eu - émanant de justiciables souhaitant principalement donner un écho à leur opinion sur la gestion de la crise. Comme cela a pu être relevé,

73 CE, ord., 22 mars 2020, Syndicat jeunes médecins, préc.

74 C. Malverti et C. Beaufils, «Le référé en liberté », préc.

75 P. Cassia, « Le Conseil d'État et l'état d'urgence sanitaire : bas les masques ! », blog de l'auteur hébergé par Mediapart, 11 avr. 2020 
le juge des référés doit prendre garde à la « confusion des rôles ${ }^{76}$ » et ne pas se mettre à juger l'opportunité des décisions prises par les autorités politiques.

La juridiction administrative s'est construite sur cette saine compréhension du rôle qui est le sien dans la séparation des pouvoirs : elle a su faire preuve d'audace pour s'affirmer comme un garant incontournable de la légalité et des libertés ${ }^{77}$, mais aussi d'une sage prudence qui lui a permis d'affermir petit à petit sa légitimité. Mais elle doit aussi continuer les efforts entrepris dans le but d'être mieux comprise par les citoyens, qui ont parfois du mal à situer le Conseil d'État dans le jeu institutionnel et à prendre conscience qu'il est un juge à leur service. Cette problématique, d'ailleurs, n'est pas propre à l'ordre administratif ${ }^{78}$. Les efforts entrepris pour rendre plus accessibles et compréhensibles les décisions du juge administratif vont dans ce sens. La juridiction doit aussi communiquer de manière pédagogique : c'est ce qu'elle a tenté de faire pendant l'état d'urgence sanitaire, en publiant des communiqués sur toutes les décisions importantes. Le président de la section du contentieux et moimême nous sommes par ailleurs exprimés à de nombreuses reprises dans la presse. Ces exercices de communication comportent naturellement des risques, mais je reste persuadé que le Conseil d'État doit continuer dans cette direction car il sera seul responsable, en définitive, si ceux qu'il sert ne le comprennent pas.

$$
* * *
$$

Voici donc, Mesdames et Messieurs, chers amis, comment le Conseil d'État français a fait face à la crise sanitaire. Je sais que les méthodes du juge et les modalités de son contrôle sont trop liées à l'histoire et à la culture d'un pays pour qu'il puisse prétendre détenir les clés de la justice universelle. Pascal l'a bien dit : « Vérité en-deçà des Pyrénées, erreur au-delà ». Et c'est précisément car nous sommes différents que nous avons à apprendre les uns des autres et que notre coopération bilatérale est si précieuse. Je forme donc le vœu que ces journées soient stimulantes et constituent une nouvelle fois l'occasion de nous enrichir mutuellement.

Je vous remercie.

76 J.-M. Sauvé, « Bilan de quinze années d'urgence devant le juge administratif », discours du 26 juin 2015, disponible sur le site internet du Conseil d'Etat

77 Il n'est qu'à citer quelques décisions emblématiques telles que CE 17 février 1950, Ministre de l'agriculture cl Dame Lamotte, $\mathrm{n}^{\circ} 86949$; CE 19 octobre 1962, Canal, Robin et Godot, $\mathrm{n}^{\circ} 58802$; ou, plus récemment, CE, Ass., 10 juillet 2020, Association Les amis de la Terre France et autres, $\mathrm{n}^{\circ} 428409$

78 Rapport de la Commission de réflexion sur la réforme de la Cour de cassation, p. 129 et $\mathrm{s}$. 


\section{STATE COUNCIL IN THE FACE OF THE COVID-19 HEALTH CRISIS}

In this paper, the author analyzes the way in which the French State Council dealt with the health crisis. Even though the court's methods and means of its control are too narrowly connected to the history and culture of the country to conclude that this institution holds key universal justice, the authors by referring to Pascal ("Truth below the Pyrenees, elsewhere error") concludes that, due to our differences, we must learn from each other which is why bilateral cooperation is so valuable.

Key words: France, State Council, Covid-19 health crises 